\title{
The Indian diabetic risk score- To Nip in the Bud
}

\author{
Dr. A. J. Manjula Devi, Dr. A. Jamuna Rani (Corresponding Author) \\ Prof and H.O.D. Dept. of Biochemistry, Sree Balaji Medical College and Hospital...\#7,Works Road,Chromepet \\ Chennai-44.Tamil Nadu
}

\begin{abstract}
:
Introduction: The International Diabetic Federation (IDF) Diabetic Atlas Fifth edition reports that half of the diabetic patients are undiagnosed. India has earned the reputation of being termed the Diabetic Capital of the world. Diabetes, which was once prevalent only among adults, is now found commonly in children due to change in lifestyle and imbalanced eating habits. More and more young adolescents are falling prey to the disease. So mass awareness and screening programs are the need of the hour.

Material \& Methods: This study was carried among 100 I M.B.B.S students of Sree Balaji Medical College. Indian Diabetic Risk score (IDRS) questionnaire was used to score the students. Students in the risk score above $60 \%$ were advised to undergo Fasting and Post prandial blood sugar levels.
\end{abstract}

Results: Only one student showed high risk whereas the study revealed that $51 \%$ of student lacked exercise in their schedule.

Key words: Type 2 Diabetes, Indian Diabetic Risk score IDRS, medical students.

\section{Introduction}

A recent by Mohan et al study has shown how increasing awareness and empowerment of community can possibly help in the prevention of diabetes and other non communicable disorders. ${ }^{(1)}$ Moreover, the 'fast food culture' which is widespread in our cities and towns is also a major cause of the diabetes epidemic. The 'fast-foods' that are fat and calorie rich are easily available in various food outlets. As a majority of the immigrants in Chennai depend on these unhealthy 'junk' foods, this may be a major factor in the rising prevalence of diabetes and cardiovascular diseases in urban slums. One point worth mentioning is that diabetes can no longer be considered as a disease of the rich. The prevalence of diabetes is now rapidly increasing among the poor in the urban slum dwellers, the middle class and even in the rural areas. This is due to rapid changes in physical activity and dietary habits even among the poorer sections of the society. Unfortunately the poor diabetic subjects delay taking treatment leading to increased risk of complications ${ }^{(2)}$ Non Communicable Diseases due to lack of a clear etiological agent is heavily dependent on identifying and tackling risk factors. The risk factors like age, gender,family history are non-modifiable while others like smoking, diet, physical activity, hypertension, diabetes etc are modifiable. ${ }^{(3)}$

\section{Aims and objectives}

This study was aimed to create awareness among the students about Type 2 Diabetes mellitus and also help identify the high risk population as well as drive home the message about incorporating exercise in regular lifestyle especially among the adolescent students.

\section{Materials and Methods}

The study was a cross sectional study. It was conducted in the department of Biochemistry Sree Balaji Medical College and Hospital. This study was approved by the Institutional Ethics committee. The period of study was June 2012. The I M.B.B.S students were briefed about the purpose of the study. The sampling method was convenient sampling method. The study was based on the Indian Diabetic Risk score questionnaire. The IDRS is a very convenient and simple method to study the risk for Diabetes among the Indian population. ${ }^{(4)(5)(6)}$ Students with a score more than $60 \%$ were advised to have their Fasting Plasma Glucose (FPG) and Post Prandial Plasma glucose (PPPG).The FPG \& PPPG was assayed using the Glucose Oxidase Peroxidase (GOD POD) method. Results: Out of 100 students who participated in the study only one student had high risk above 60. This one student when advised FBS and PPBS showed normal values. We were surprised to see that $51 \%$ of students did not incorporate exercise in their schedule. 16\% had mild exercise routine whereas $19 \%$ had moderate exercise in their routine.

\section{Discussion}

A similar study carried out among students in Maharashtra has reported that no student had strenuous physical activity in their schedule and only $10 \%$ had minimal physical activity. ${ }^{(7)}$ Our study has revealed that 
girls had higher percentage of moderate diabetes risk when compared with boys .The increased number of girls in the moderate risk group was mainly due to lack of exercise. We saw that boys indulged in strenuous exercise as playing Volley ball, cricket or football whereas very few girls had strenuous exercise in their schedule.

\section{Conclusion}

As a result of this study we have identified that lack of exercise was the main risk factor among this adolescent population. We have had a detailed counseling on the essence of exercise in the schedule of these budding physicians. Statistics says half the people with diabetes are undiagnosed as diabetes is a "silent"disease.

${ }^{(8)}$ This study should be conducted among various groups of adolescents and young adults to bring about mass awareness and prevention of diabetes. Our study has also helped carry the message across to a part of the society through our budding physicians.

\section{Acknowledgement}

We thank the 100 students volunteers of the first M.B.B.S (2012-2013) batch of students .

\section{References}

[1]. Mohan D, Raj D, Shanthirani CS, Datta M, Unwin NC,Kapur A, et al. Awareness and knowledge of diabetes in Chennai - the Chennai Urban Rural Epidemiology Study (CURES-9). J Assoc Physicians India 2005; 53: 283-7.

[2]. Ramachandran A, Snehalatha C, Vijay V, King H. Impact of poverty on the prevalence of diabetes and its complications in urban southern India. Diabet Med 2002;19: 130-5.

[3]. Vardhan A, Adhikari Prabha MR,Kotian Shashidhar M,Saxena N,Gupta S,Tripathy A.The value of the Indian Diabetes Risk score as a tool for reducing the risk of diabetes among Indian Medical Students.J.Clin Diagn Res 2011;5:718-20

[4]. Shashank R JoshiIndian Diabetes Risk Score JAPI • VOL. 53・SEPTEMBER 2005

[5]. Prabha Adhikari, Rahul Pathak, Shashidhar Kotian -Validation of the MDRF - Indian Diabetes Risk Score (IDRS) in another South Indian Population through the Boloor Diabetes Study (BDS) JAPI • July 2010 • Vol. 58

[6]. V Mohan, R Deepa, M Deepa, S Somannavar, M Datta- A Simplified Indian Diabetes Risk Score for Screening for Undiagnosed Diabetic Subjects JAPI • VOL. 53 • SEPTEMBER 2005.

[7]. Pranita Ashok, Jayshree S.Kharche, Aniruddha R.Joshi -Evaluation of Risk for Type 2 Diabetes Mellitus in Medical students using Indian Diabetic Risk score-Indian Journal of Medical sciences, Vol 65,No.1,January 2011.

[8]. Mohan V, Deepa M, Deepa R, Shanthirani CS, Farooq S, Ganesan A, Datta M. Secular trends in the prevalence of diabetes and impaired glucose tolerance in urban south India - the Chennai Urban Rural Epidemiology Study (CURES-17). Diabetologia 2006; 49:1175-1178.

Table 1

\begin{tabular}{|l|l|l|l|l|}
\hline Category & Risk score & Male & Female & $\%$ Total \\
\hline Low risk & $<30$ & 39 & 18 & $57 \%$ \\
\hline Moderate risk & $30-50$ & 9 & 33 & $42 \%$ \\
\hline High risk & $>60$ & 1 & nil & $1 \%$ \\
\hline
\end{tabular}

Table 2 Indian Diabetic Risk score

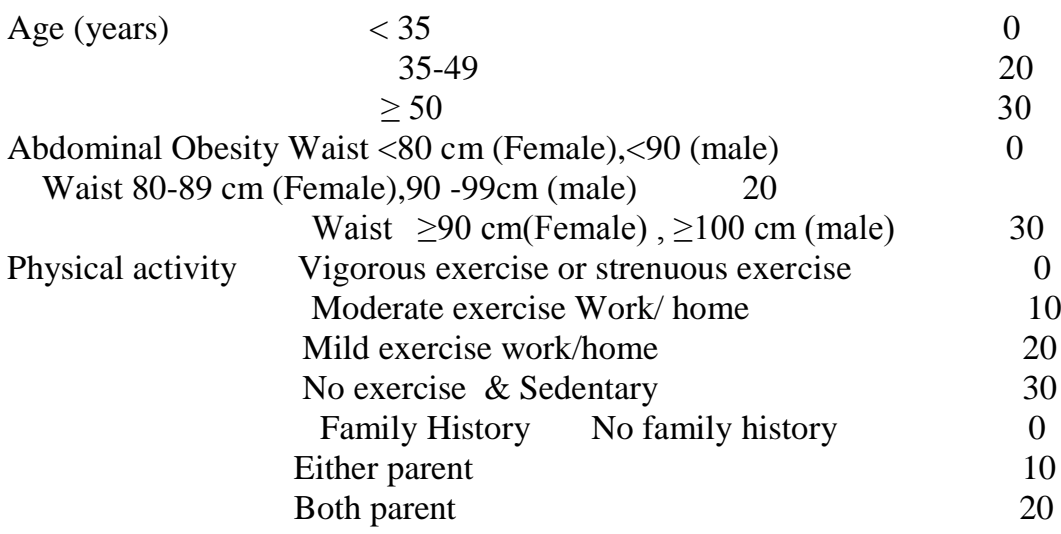

Maximum score 